tion par un colibacille dont la virulence serait particulièrement accrue en été. Cette mammite est parfois observée, durant plusieurs années, dans la même étable.

\title{
BIBLIOGRAPHIE.
}

[1] Lucet. Recueil de médecine vétérinaire, 1889, p. 423, et De la congestion des mamelles et des mammites aiguës chez la vache, 1891.

[2] P. Leblanc. Les maladies des mamelles chez les animaux domestiques (1901).

[3] Tobrassen. Ueber eine ligenthümliche Form der Eüterentzundung der Kühe. Maaned. f. Dyrlaeger, VIII, 94, 51.

[4] Villumsen. Eine besondere Form der Eüterentzundung. Maaned.f. Dyrlaeger, VIII, p. 243.

[5] Chapron. Rapports de la fièvre vitulaire et de la pseudo-paraplégie après mammite. Revue de pathologie comparée, 1924, p. 763.

[6] Quintard. La mammite pseudo-paraplégique des vaches laitières. Thèse pour le doatorat vétérinaire, Toulouse, 1930.

[7] Castellani et Chalmers. Sur la classification de certains groupes de bacilles aérobies de l'intestin humain. Annales de l'Institut Pasteur, 1920.

[8] Lesbouyries et Adam. Diagnostic étiologique des mammites streptococcique et staphylococcique de la vache. Bulletin de l'Académie vétérinaire, 19 mai 1932.

\section{L'INFECTION LATENTE DE LA IMAMELLE ET SES RÉVEILS. LES MOYENS DE LA DÉPISTER}

\author{
par CH. PORCHER \\ Docteur ès Sciences physiques.
}

(Fin.)

\section{LA DÉTERMINATION DE L'ESPÉCE MICROBIENNE PRINCIPALE.}

Nous disons principale, puisque très souvent une association apparaît, surtout lors des réveils. Lorsque l'infection est latente, une seule espèce domine en général.

Il y a le plus grand intérêt, avons-nous déjà dit, à déterminer l'espèce microbienne qui fait le fond de l'infection. Lorsque nous voyons en face les uns des autres des microbes aussi différents que le streptocoque, agent plutôt banal, si souvent rencontré et nocif cependant, les Brucella, var. abortus et melitensis, dont le caractère pathogène indéniable pour celui-ci, s'affirme pour celui-là et le $B$. tuberculosis, il est indispensable qu'un diagnostic précis soit fait.

Les lois sanitaires interviennent et la lutte contre la tuberculose exige que toutes ses formes soient dépistées et éliminées.

Nous avons plutôt en vue, aujourd'hui, le streptocoque, mais dans cette conférence, véritable revue générale de la question, il n'était pas possible de laisser de côté les autres microbes qui interviennent pour déterminer les formes un peu différentes de 
l'infection latente. Ce n'est pas le moment d'exposer toutes les méthodes auxquelles il faut recourir pour déterminer avec précision la nature microbienne de l'infection latente de la mamelle : colorations, ensemencements, inoculations sont d'ailleurs connus.

$$
* * *
$$

Nous en avons fini avec l'examen, rapide d'ailleurs, auquel nous avons procédé pour caractériser les laits malades et les laits infectés sous toutes les formes, depuis les plus bénignes, voisines de l'état normal et qui se rapprochent presque de lui, jusqu'aux plus graves.

Si nous restons dans la zone des laits apparemment normaux, laits tous d'infection latente, nous trouverons quelque intérêt à faire la récapitulation de toutes les données sur lesquelles nous avons insisté plus ou moins dans les pages qui précèdent.

Un lait d'infection latente, toujours apparemment normal, mais dont les altérations dans la composition chimique, biochimique et cytologique sont déjà notables, - et c'est en général à ces laits que vous aurez affaire dans la pratique, - se distingue du lait normal :

A. Dans les molécules élaborées vraiment caractéristiques de la sécrétion lactée :

$1^{\circ}$ Par une diminution du lactose. Celle-ci est le fait de :

a) Du trouble apporté dans la sécrétion par l'infection;

b) De celui qu'entraîne le processus de rétention, toujours observable en de pareilles circonstances.

A la diminution du lactose correspond :

Une diminution de l'indice de réfraction du sérum $(n)$.

$2^{\circ}$ Une diminution de la caséine, plus exactement du caséinate de calcium. De ceci résulte que, sous l'action de la présure, le coagulum obtenu sera moins consistant, il sera mou, fragmenté et le sérum, laiteux.

Mais d'autres facteurs interviennent pour expliquer le mauvais emprésurage. Ce sont: l'augmentation des protéines non vraiment caractéristiques de la sécrétion lactée, l'albumine et surtout la globuline.

$3^{\circ}$ Une diminution et même une disparition complète de l'acide citrique qui existe dans le lait sous forme de citrates.

B. Dans les éléments également caractéristiques de la sécrétion lactée, mais qui ne sont pas à proprement parler d'un caractère spécifique, par :

La diminution du taux des phosphates.

L'anion phosphorique, de même que les cations potassique et 
calcique, diminue lors de l'infection latente. Cette diminution entraîne :

Une diminution de l'acidité titrable, acidité potentielle, ainsi que de l'acidité actuelle déterminée potentiométriquement. Les laits d'infection latente seront done des laits alcalins. Leur $p \mathrm{H}$ s'approchera de 7 et souvent dépassera 7. Les laits d'infection latente se caractérisent ainsi par une diminution dans leurs cendres de l'acide phosphorique, de la chaux et, disons aussi, de la magnésie.

\section{Dans les éléments non élaborés de la sécrétion lactée.}

$1^{\circ}$ Molécules protéiques : albumine et globuline. Le taux de l'albumine dans le lait de vache oscille autour de 3,5 à 4,5 par litre, celui de la globuline autour de quelques centigrammes. Le lait d'infection latente se caractérise par une augmentation de ces protéines, beaucoup moins du côté de l'albumine que du côté de la globuline, nous serions tenté de dire presque uniquement du côté de celle-ci. Il en résulte que l'emprésurage paresseux, défectueux qui dépend, pour une part, d'une diminution du taux de la caséine, devrait être mis également, pour une autre part, au compte de l'augmentation de la globuline, colloïde éminemment protecteur.

$2^{\circ}$ L'augmentation de l'azote non protéique. L'azote non protéique, qui constitue une partie importante de ce qu'on appelle l'indosé dans le lait normal s'exagère considérablement dans l'infection, comme dans la rétention d'ailleurs, et peut atteindre un taux notable, plusieurs grammes au litre.

$3^{\circ}$ Molécules salines banales. Sous cette acception nous rangeons quelques matières salines, telles que les chlorures, les sulfates et les bicarbonates. Il y a lieu de noter au cours de l'infection latente :

a) Une augmentation de l'acide sulfurique, par conséquent des sulfates.

b) Une augmentation de la soude et de l'acide carbonique sous forme de bicarbonate et peut-être, dans les formes très alcalines, d'un peu de carbonate neutre de sodium. En même temps donc que disparaissent les éléments d'acidité, comme les phosphates potassiques, le mono - en tête, nous voyons apparaître, se substituer à eux peut-on dire, des éléments d'alcalinité, tels que le bicarbonate et le carbonate sodiques.

c) Une augmentation du chlorure de sodium, conformément à la loi de Ch. Porcher. Cette augmentation marche de pair avec la diminution du lactose. Il en résulte :

๙) Une saveur salée du lait un peu spéciale, comme nous l'avons vu, parce qu'elle est le fait de l'association de deux 
processus inverses: une augmentation du chlorure de sodium, une diminution du lactose, dont la saveur sucrée est, il est vrai, peu marquée, mais qui n'en contribue pas moins à donner au lait normal son goût agréable.

§) Une augmentation de la conductibilité du lait, c'està-dire une diminution de la résistance $(r)$.

Si nous récapitulons toutes les conséquences des trois paragraphes principaux sous lesquels nous avons rassemblé, en les ordonnant, les modifications de la composition du lait qui sont le fait de l'infection latente, nous dirons que, analytiquement :

A. Dans les laits infectés, l'extrait dégraissé est moindre.

B. L'extrait dégraissé délactosé est plus élevé, paree que le taux du lactose diminue en général assez fortement.

D. Modifications cytologiques. Ce sont surtout les phagocytes que nous visons. Dans l'infection latente :

a) Leur nombre s'accroît en valeur absolue ;

b) Le pourcentage des polynucléaires phagocytes neutrophiles est considérable. Il varie de 75 à $98 \%$ sur l'ensemble des leucocytes.

E. Les modifications biochimiques. Nous nous limiterons ici à celles qui ont trait aux diastases et encore entre celles-ci faisonsnous un choix et n'envisageons-nous que l'une d'entre elles, la principale, la catalase, qui est sécrétée presque entièrement par les phagocytes, notamment les polynucléaires. Elle est done vraiment un produit de l'inflammation et son quantum ou, plus exactement, la valeur de l'indice catalasimétrique, donne en quelque sorte la mesure de l'intensité de l'infection.

Ce qui fait l'intérêt d'une pareille récapitulation, c'est le parallélisme frappant de toutes les données que nous avons relevées en l'établissant.

Depuis longtemps, nous avons appelé l'attention sur l'harmonie de la composition du lait à l'état normal. Mais il en est une également pour l'état pathologique.

A la base de l'étude de toute cellule vivante, disions-nous dans l'avant-propos de notre thèse de doctorat ès sciences physiques, se trouve inéluctablement cette donnée que son fonetionnement ne saurait être faussé d'une manière quelconque. Si celui-ci est troublé, la cellule réagit, mais elle le fait dans une direction déterminée. A l'harmonie du fonctionnement normal s'ajoute ou plutôt se substitue l'harmonie du fonctionnement anormal ou pathologique et nécessairement la composition du produit sécrété doit être le 
reflet de eette dernière. Les rapports des divers composants ne sont plus les rapports de l'état normal, mais eux aussi ne sont point queleonques et nous devons les expliquer.

Nous venons de voir que toutes les données analytiques qu'un lait malade peut fournir, qu'elles relèvent de la chimie, de la chimie-physique, de la biochimie ou de la cytologie, s'enchaînent étroitement, si bien même que, le plus souvent, l'une d'entre elles pourrait suffire pour porter un premier jugement sur un lait malade.

Un lait de saveur salée a nécessairement un taux faible en lactose, un indice de réfraction abaissé, une résistance affaiblie. Il sera alcalin, il coagulera mal par la présure, son indice catalasimétrique sera élevé et, couronnant le tout, nous trouverons le plus souvent qu'il contient des streptocoques de la mammite. Mais sur ce dernier point, nous devons être moins affirmatifs. Il peut renfermer toute autre espèce microbienne, comme le bacille tuberculeux ; les modifications de sa composition que nous venons de voir n'en resteront pas moins les mêmes.

L'exposé que nous venons de vous faire peut être considéré comme une synthèse d'acquisitions relevant des disciplines différentes auxquelles la biologie emprunte. Il nous faut choisir maintenant celles qui simplement, rapidement et sûrement dans le cadre de votre activité professionnelle vous permettront d'établir un diagnostic d'où découleront toutes les conséquences possibles au double point de vue de la thérapeutique et de la police sanitaire.

Supposez que les circonstances fassent que vous soyez appelé dans une étable où sévit de la mammite : l'infection s'est réveillée chez quelques-uns des sujets, mais les autres paraissent encore indemnes, leurs mamelles et le lait sont apparemment normaux. Tout au plus a-t-on pu constater, à côté des bêtes qui-présentaient des signes cliniques manifestes d'une infection de la mamelle, pour laquelle d'ailleurs on vous consulte, quelques diminutions de rendement chez les animaux voisins, non sur les 4 quartiers, mais sur un ou deux d'entre eux.

Vous pouvez être appelé, et c'est un second aspect de votre intervention sur le même terrain, par des propriétaires attentifs qui n'ignorent rien des désastres que certains réveils de l'infection latente de la mamelle peuvent produire dans leur cheptel. Vous vous trouvez done placé, dans un cas comme dans l'autre, devant la maladie invisible d'un seul organe d'un animal dont la santé générale est excellente. Vous procéderez à l'examen clinique de la mamelle, comme vous en avez l'habitude, et vous trouverez peu de chose, quelquefois rien. Peut-être par un doigté que la grande pratique vous aura permis d'acquérir, sentirez-vous dans certains quartiers plutôt 
que dans d'autres, notamment dans les postérieurs, quelques noyaux diffus, plus ou moins fondus dans le reste du parenchyme normal, ou assez bien circonscrits. Pas de retentissement ganglionnaire ; les ganglions rétro-mammaires sont d'un volume normal et non indurés. La carence de la réaction des ganglions rétro-mammaires ne saurait toutefois vous permettre d'éliminer ipso facto une infection mammaire, infection plutôt tuberculeuse, car les ganglions réagissent bien surtout devant le bacille tuberculeux. Lorsque l'infection est de nature streptococcique ou brucellique, la réaction ganglionnaire est négligeable. Elle est même le plus souvent absente.

Bref, l'inspection de la mamelle ne vous ayant positivement rien donné de net, vous faites procéder à la traite séparée des 4 quartiers.

Le lait est goûté, il a une saveur salée ou il n'en a pas. La saveur salée vous indique incontestablement une infection du quartier correspondant. Ce lait salé a une réaction alcaline que vous pourrez déceler séance tenante par l'épreuve de l'alizarol.

Il a un indice catalasimétrique élevé dont vous pourrez confier la mesure à un laboratoire, à moins que chez vous, et vous savez que le matériel n'est pas encombrant et que les manipulations sont simples, vous fassiez vous-même ou fassiez faire la réaction de la catalase.

Enfin vous pouvez centrifuger le lait sur place avec une petite trousse disposée à cet effet, en utilisant les tubes de TrommsDorfF, à moins que, encore une fois, vous ne le fassiez chez vous. Nous n'exigeons rien ici qui puisse vous prendre beaucoup de temps ou vous éloigner de vos préoccupations habituelles. Toutes ces manipulations sont simples et vous en faites couramment qui sont plus compliquées.

Certaines précautions doivent cependant être prises par vous. Les prélèvements de lait ne doivent pas être quelconques. Placés dans une région d'élevage où vous êtes susceptible d'être appelés fréquemment pour le cas qui nous occupe, il importe que vous ayez une réserve de petits flacons propres, stériles autant que possible, munis d'un bouchon, que vous ouvrirez au moment même de faire le prélèvement et que vous reboucherez immédiatement après. Quelquefois on peut substituer à ces flacons des tubes à essais courts et épais, moins fragiles, munis eux-mêmes d'un bouchon pour l'expédition des échantillons dans un laboratoire où l'on procédera à des recherches plus minutieuses pour lesquelles vous n'êtes pas suffisamment outillé, notamment la détermination de l'espèce microbienne et l'examen cytologique.

Si l'examen de la mamelle, en vue de dépister l'infection latente, devait se ramener à un simple constat, si l'établissement du diag- 
nostic ne devait pas être suivi de conséquences, cette conférence n'eût point été faite. Les développements dans lesquels nous sommes entré, vous ont montré, nous le pensons du moins, qu'il est utile de suivre pour vous la route que nous avons indiquée.

L'examen de la mamelle, en état d'infection latente, ne doit pas être stérile. Le diagnostic fait, - et pour qu'il soit complet, vous devez vous appuyer sur le laboratoire, - vous devez en prévoir les conséquences.

Mettez le propriétaire en garde contre les réveils possibles et particulièrement graves au moment des épidémies de fièvre aphteuse. Appelez son attention et celle de ses employés sur les moindres signes qui d'habitude leur échappent parce qu'ils ne regardent point ou ne savent point regarder les ehoses. Faites-vous appeler au moindre réveil, à la plus petite manifestation clinique. L'avenir économique d'un animal, - et quand c'est un animal de prix, a fortiori peut dépendre de la négligence de l'homme qui le soigne d'habitude.

Dans un troupeau important, fait de têtes de valeur, troupeau d'élevage ou de haute production laitière, des "visites dè santé " de la mamelle, visites régulières, nous semblent indiquées.

Nous pensons, pour en revenir à l'exemple que nous avons eu sous les yeux au Chili, que les désastres causés par une seconde épidémie de fièvre aphteuse à 3 mois de la première eussent été moindres si de semblables visites, indiquées plus que jamais en raison de la première épidémie qui avait éclaté déjà, avaient été faites.

Quand nous vous demandons de faire appel au laboratoire, c'est parce que vous vous trouverez devant des infections numériquement très différentes. Attention, comme nous l'avons dit, à ces quartiers dont l'envahissement microbien est notable. Ce sont les premiers candidats aux réveils de l'infection. Ceux-ci se manifesteront également au moment de "l'égouttage", lorsqu'on mettra la mamelle " au sec».

Les mammites à cette époque ne sont pas particulièrement graves et se résoudront assez vite, mais elles indiquent qu'il y a là une flore abondante et assez vigoureuse dont il faut surveiller les manifestations.

Recommandez bien à vos clients d'éviter, pendant les épidémies de fièvre aphteuse lors des infections et générales qui frappent l'animal, de ne pas espacer les traites, de ne pas les faire sans application. Bien au contraire, insistez pour que la traite soit toujours faite aussi complètement que possible, même quand la sécrétion diminue beaucoup.

Et, pour ne rien omettre, répondez à l'argument de la présence d'aphtes sur les trayons, de la sensibilité de l'animal et de sa défense, en recommandant, d'une part, la douceur, et, d'autre part, l'emploi 
de graisses consistantes que le commerce a mis à la disposition des propriétaires, dans le but de permettre la traite dans des circonstances généralement assez difficiles. La conservation d'un animal, avec la sauvegarde de sa mamelle en son entier ou de plusieurs de ses quartiers, dépend de ces soins qu'on n'a pas l'habitude de prendre parce qu'on est dans l'ignorance, pensons-nous, des conséquences funestes de leur omision.

Il est évident que les "visites de santé " mammaires sont d'un grand intérêt et d'une utilité que nous ne pouvons plus aujourd'hui discuter. Mais il faudra beaucoup de persuasion pour en faire prendre l'habitude. Le producteur plus ou moins bien éclairé, plutôt mal que bien, préférant comme beaucoup le moindre effort, ne s'émeut que lorsqu'il est placé devant la gravité d'un eas souvent irréparable.

La maladie invisible, comme l'est l'infection latente de la mamelle, demande qu'on intervienne avant qu'elle ne devienne visible. C'est beaucoup exiger et c'est prévoir dans la mentalité des modifications que la paresse humaine et la négligence ne semblent pas rendre prochaines.

Comme nous l'écrivait un de nos confrères qui avait bien voulu nous consulter pour un cas de réveil d'une infection mammaire et à qui nous avions répondu en lui donnant quelques conseils, "notre plus grand adversaire, c'est le propriétaire, qui cependant devrait être intéressé à la bonne santé de son bétail. Il ne nous appelle que lorsqu'il n'y a souvent plus rien à faire, quand la situation est plus ou moins compromise et qu'il est difficile de revenir à l'état antérieur.

" Si certaines manipulations doivent être faites par lui sur notre conseil, car notre elientèle nous met souvent dans l'impossibilité d'être à une heure fixe pour certains traitements, il ne fait rien. "

Ce qui ajoute aux difficultés de la question, c'est son absence de thérapeutique. Des traitements spécifiques, des vaccins ont été recommandés, mais il semble bien qu'aucun n'ait encore donné de résultats positifs.

Traiter l'infection latente alors que la mamelle est moyennement envahie par une flore qui peut se réveiller, mais ne le fera pas fatalement, e'est intervenir dans des circonstances qui étonneront fort le propriétaire. Il n'acceptera que difficilement qu'on vienne soigner des animaux qui ne sont pas malades, dira-t-il. Votre intervention ne se comprendra à ses yeux, et encore ! que pour les quartiers que le laboratoire vous aura désignés comme fortement infectés. Dans tous les cas, vos conseils restent. Leur utilité ne peut pas être tournée en dérision ; nous vous en avons donné des exemples.

Sans que nous ayons positivement fait des recherches sur la 
thérapeutique de la mamelle, en état d'infection, nous recommandons volontiers et nous vous demandons de l'essayer, les injections d'eau formolée tiède. Il y a déjà plusieurs années que les solutions extrêmement diluées mais tiédies aux environs de $35^{\circ}$, ont été préconisées par plusieurs confrères et, tout dernièrement, dans le traitement de certaines mammites, lors de réveils eliniquement affirmés. Nous espérons beaucoup de cette thérapeutique, simple et énergique tout à la fois. Les solutions de formol doivent être à 1 ou 2 pour 1.000 , tout au plus ; nous vous demandons de les faire sous pression après avoir détergé fortement la glande par une mulsion très soignée, puis de recommencer la traite $1 / 4$ d'heure ou $1 / 2$ heure après que la solution de formol a été injectée. Peut-être, et ce sera à vous d'en juger, faudra-t-il recommander de la recommencer le jour même et une ou deux fois les jours suivants pendant le temps que vous indiquera l'évolution de l'infection. Il y a, en l'espèce, des signes eliniques sur la valeur desquels vous êtes le seul juge.

Il y a bien des années que nous avons appelé l'attention des milieux vétérinaires sur l'importance de l'infection latente de la mamelle. Nous avions employé une expression à laquelle nous tenons plus que jamais, celle de "la police sanitaire des mammites".

Puisque la production laitière est très dépendante de la mammite, que celle-ei lui cause un préjudice considérable, que peu de sujets lui échappent, il est essentiel - la méthode curative ne donnant rien encore - de faire appel à la méthode préventive. Nous vous avons apporté aujourd'hui les moyens de la mettre en ouvre, de fixer à coup sûr votre diagnostic, de prévoir des accidents ultérieurs et souvent de les éviter.

C'est avec plaisir que nous avons vu la police sanitaire que nous demandions depuis si longtemps pour la mammite, être mise en pratique dans certains pays. C'est ainsi que dans la traduction de G. Collumbien, du 10e rapport (1928-1929) annuel de la Frise (Pays-Bas), nous lisons que régulièrement les fermes sont examinées au point de vue de la mastite infectieuse qui peut frapper leur bétail.

"Jusqu'ici, nous dit le rapport, nous n'avons pas essayé d'entamer une lutte systématique contre la mastite à streptocoques. Mais il nous semble qu'il est temps qu'un contrôle soit étendu à toutes les fabriques. Après les dix années écoulées, nous n'avons pas obtenu de progrès. Les injections de sérum et de produits chimiques n'ont pas donné les succès promis par les attestations. Dans une entrevue avec les vétérinaires pratiquant en Frise, nous aboutîmes à la conclusion que le meilleur traitement pour la mammite infectieuse était comme suit :

$1^{\circ}$ Dans le premier cas, tenir au froid et traire chaque heure; 
$2^{\circ}$ Injection immédiate par le vétérinaire de sécrétion lactée du pis malade;

$3^{\circ}$ Après 2 ou 3 jours, injection de Stock-vaccin ;

$4^{\circ} \mathrm{Si}$ c'est nécessaire, faire suivre par une injection d'autovacein.

"Le laboratoire tâchera d'avoị toujours du Stock-vaccin et d'exécuter les demandes multiples d'autovaccin.

"Pour les étables fortement infectées, nous avons appliqué les vaccinations préventives vers la fin de la période de lactation. On ne saurait trop insister sur le caractère infectieux de cette maladie. Une vache atteinte peut être considérée comme dangereuse pour les autres, et être isolée. On la traira la dernière et on anéantira sa sécrétion. ")

"En Suisse, nous dit le Laitier romand, l'inspection des fromageries, des étables a été organisée dans plusieurs cantons. Il paraît superflu de relever les précieux services rendus par l'inspection des étables. La nécessité urgente d'une inspection bien faite de l'état du fonctionnement des mamelles des vaches d'une Société de laiterie ressort de l'exemple typique suivant : il s'agissait d'une Société de fromagerie qui fabriquait de l'emmenthal. Dans le courant de l'été dernier de minutieuses inspections d'étables ont été faites chez tous les sociétaires. Ces derniers ne sont nullement opposés au principe de l'inspection, car ils la savent utile et non pas tracassière. Ils se rendent parfaitement compte que finalement la découverte de vaches à mamelle anormale leur profite directement et qu'elle rend en même temps service à la communauté, c'est-à-dire à la Société de Laiterie. Le nombre de vaches visitées a été de 426 . 15 avaient la mamelle atteinte d'une mammite déclarée ou en état de développement, chez 21 se trouvaient d'autres maladies de la mamelle, 43 livraient un lait à coagulation paresseuse et il y a là, ajouteronsnous, un signe d'infection latente. 2 livraient un lait ne coagulant pas du tout par la présure.

"Nous sommes persuadés, ditle rapporteur, que la plus grande partie des propriétaires de ces vaches n'étaient aucunement au courant de ces maladies de mamelles, pas plus que des anomalies de la production laitière.

"L'inspection des étables faites par les gens qualifiés a permis de rendre les producteurs de lait attentifs à cette anormalité qui frappait environ $20 \%$ des animaux.

"Nous pensons que les résultats de l'inspection des étables de cette Société de laiterie ne représentent aucunement un cas isolé. "

Nous n'ajouterons rien à un rapport aussi éloquent et qui plaide pour la thèse, que nous soutenons depuis si longtemps, d'un examen 
systématique des mamelles, en vue d'une police sanitaire qui éliminera de la production laitière des animaux dont la mamelle est, presque à l'insu des propriétaires, frappée par une infection plus ou moins latente qui diminue le rendement quantitativement, cela va sans dire, mais aussi qualitativement, de telle manière que l'exploitation de l'animal n'est plus rémunératrice.

La police sanitaire nous permettra chemin faisant d'éliminer également de la production laitière des animaux autrement dangereux que ceux dont la mamelle n'est envahie que par le streptocoque. Nous faisons allusion aux bêtes tuberculeuses et dont la mamelle est touchée également par le bacille spécifique.

$$
*^{*} *
$$

Notre exposé appelle également des réflexions sur les conséquences de la systématisation d'une inspection des mamelles des vaches laitières relativement au texte qui régit la production d'un lait "propre et sain".

Ce texte, - l'article 2 du décret du 25 mars 1924, - s'abrite, et on peut se demander pour quelles raisons profondes, sur la loi du $1^{\mathrm{er}}$ août 1905 relative à la répression des fraudes et nous dit, dans ses premières lignes :

"Ne peut être considéré comme lait propre à la consommation humaine :

$1^{\circ}$ Le lait provenant d'animaux atteints de maladies dont la nomenclature sera donnée par arrêté du Ministre de l'Agriculture, pris sur avis du Comité consultatif des épizooties. "

Nous verrons donc un jour publier ce texte. Nous ne pensons pas que ce soit proche, car rien n'est plus difficile que de réglementer utilement en la matière.

Tout ce que nous avons dit aujourd'hui tend évidemment à préconiser l'inspection systématique des étables en vue de l'amélioration de la production laitière. Nous trouverons des résistances sur un pareil terrain, résistances de gens mal éclairés, mais l'intérêt général nous amènera fatalement un jour à cette solution. Celle-ci réclamera des textes et l'arrêté y aura sa place. Que de circonspection, vous le devinez, il faudra pour en fixer les termes. D'ores et déjà, on peut prévoir que nous aurons devant nous les intransigeants et ceux qui composent. Il en est qui voudront englober toutes les mammites dans les mêmes dispositions légales, déclarer impropre à la consommation le lait de mammite streptococcique, aussi bien que celui de la mammite tuberculeuse. Ce serait une erreur grave à notre avis, parce que la nocivité de tous les laits de mammite streptocoecique n'est point encore démontrée. Si nous réclamons la police sanitaire des mammites du point de vue économique autant 
que du point de vue hygiénique, e'est en vue de préserver le eheptel national contre une affection à laquelle il paye un trop lourd tribut. Il se trouve qu'en même temps nous luttons sur le terrain hygiénique. Rien n'est plus naturel, ear il n'est pas possible de dissocier les deux points de vue. Mais il faut se garder des conséquences extrêmes, de considérations par trop théoriques. Rien n'est plus lamentable que de dire : "Périssent les eolonies plutôt qu'un principe. " En politique sanitaire, comme en politique tout court, rien de plus désastreux que semblable formule, qui frise l'imbécillité. A en poursuivre l'application, on compromet la solution du bon sens. C'est se faire le serviteur d'une mauvaise cause.

Evidemment, rien n'est plus difficile pour répondre au premier paragraphe de l'article 2 que d'établir la liste prévue par l'arrêté ministériel pour l'application de cet article. On peut déjà prévoir où se trouve la vérité. La sertir dans un texte qui ne soit pas un nid à chicanes et à interprétations tendancieuses est autrement délicat.

On y arrivera certainement, paree que les recherches entreprises en tous pays par tant de travailleurs sur la question qui a fait l'objet de cette conférence nous permettront de jalonner la route qui nous mènera au but.

Les solutions d'aujourd'hui sont celles de problèmes qui ont été posés il y a bien des décades. Il en sera de même de celui-ci. Nous ne nions pas les difficultés, nous les avons fait entrevoir. Un dispositif trop absolu inquiéterait et il doit être évité.

Quoi qu'il advienne, on ne doit pas perdre de vue que l'amélioration de la production laitière, à laquelle les pouvoirs publics, défenseurs naturels de l'intérêt général, se doivent d'apporter tous leurs soins, ne sera jamais ce qu'elle doit être tant qu'on négligera l'examen systématique des mamelles.

Il est du plus grand intérêt pour les vétérinaires de bien se pénétrer des notions que nous avons développées devant vous aujourd'hui. Défenseurs de l'élevage, il vous est indispensable de porter vos efforts plus que vous ne l'avez fait jusqu'ici sur le dépistage des mammites, qui causent à la production laitière un préjudice considérable. Nous vous avons montré qu'il n'est possible que par la mise en œuvre de disciplines différentes, dont l'essentiel, pour la grande pratique, a été puisé dans certaines acquisitions ehimiques et biochimiques qui s'en inspirent. Votre culture générale et vos études vous mettent très à même de répondre à ce qu'on attend de vous en cette matière (1).

(1) La bibliographie de ce travail paraîtra ultérieurement. 